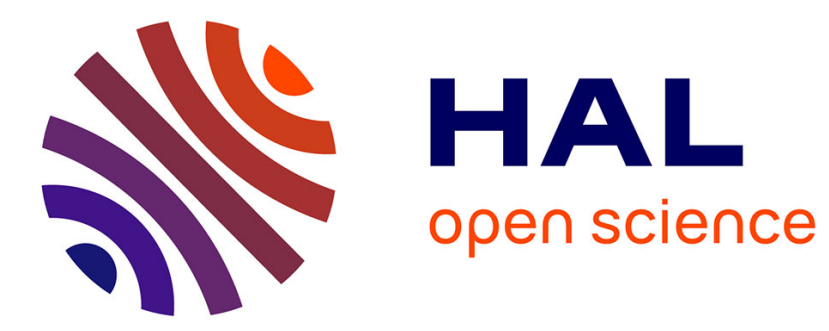

\title{
De la guerre au maintien de la paix : le nouveau business mercenaire
}

\author{
Richard Banégas
}

\section{To cite this version:}

Richard Banégas. De la guerre au maintien de la paix: le nouveau business mercenaire. Critique Internationale, 1998, 1 (1), pp.179-194. 10.3406/criti.1998.1365 . hal-01010390

\section{HAL Id: hal-01010390 \\ https: / hal-sciencespo.archives-ouvertes.fr/hal-01010390}

Submitted on 19 Jun 2014

HAL is a multi-disciplinary open access archive for the deposit and dissemination of scientific research documents, whether they are published or not. The documents may come from teaching and research institutions in France or abroad, or from public or private research centers.
L'archive ouverte pluridisciplinaire HAL, est destinée au dépôt et à la diffusion de documents scientifiques de niveau recherche, publiés ou non, émanant des établissements d'enseignement et de recherche français ou étrangers, des laboratoires publics ou privés.

\section{(이)(\$)}

Distributed under a Creative Commons Attribution - NonCommercial - NoDerivatives| 4.0 


\title{
De la guerre au maintien de la paix
}

\section{le nouveau business mercenaire}

\author{
par Richard Banégas
}

\begin{abstract}
If it take no success, it is done by their owne beddes. It is but the attempt of private gentlemen, the State suffers noe losse, noe disreputation. If it take success, they are yours subjects, they doe it for your service, they will lay at your Majesty's feet and interess your Majesty therein. Plaidoyer d'un conseiller du Roi James Ier d'Angleterre (1603-1725) en faveur de la Compagnie de Virginie ${ }^{1}$.
\end{abstract}

\section{e}

boussé par un scandale politico-diplomatique mettant en cause ses liens avec le milieu du mercenariat : la presse révélait que le Foreign Office avait eu recours aux services d'une compagnie privée de sécurité, Sandline International, pour aider les forces nigérianes de l'ECOMOG à restaurer Ahmed Tejan Kabbah, le Président démocratiquement élu de Sierra Leone, qui avait été renversé par un coup d'État en 1997. Des livraisons d'armes - en partie financées par des compagnies minières - auraient été effectuées par le canal de cette société de mercenaires au mépris d'une résolution de l'ONU, votée avec l'appui britannique, interdisant les ventes de matériel militaire aux acteurs du conflit.

Cette affaire illustre une tendance récente selon laquelle les conflits « de basse intensité » sont de plus en plus gérés par des firmes multinationales de sécurité sur lesquelles la « communauté internationale » se décharge (Hibou 1998) de ses 
velléités d'intervention. Sur le continent africain se développe ainsi un nouveau type de mercenariat qui profite des bouleversements stratégiques de l'après-guerre froide et des opportunités financières de la globalisation. De nouveaux condottieres s'affirment comme de véritables entrepreneurs transnationaux de la guerre. Ils cherchent à conquérir une légitimité et à diversifier leurs activités et utilisent à cette fin des stratégies de communication élaborées et un discours tout imprégné de l'idéologie néolibérale en vogue chez les bailleurs de fonds. Et, de fait, ils bénéficient d'une attention croissante des grandes puissances et des instances multilatérales qui, de plus en plus réticentes à intervenir en Afrique, sont tentées de sous-traiter au privé les opérations de maintien de la paix. De la privatisation de la guerre, qui est déjà d'actualité en bien des points du globe, va-t-on passer ainsi à la privatisation progressive du peacekeeping ? Le rôle croissant de ces sociétés de sécurité est-il un signe de la démission des États ou une nouvelle modalité de la souveraineté ? Et les modes d'accumulation de ces entrepreneurs, en particulier dans le domaine de l'exploitation minière, constituent-ils un « retour » à l'économie concessionnaire et préparent-ils de nouveaux conflits mercantilistes entre acteurs privés ?

\section{L'hydre mercenaire : un « empire transnational »?}

Les nouveaux condottieres prospèrent aujourd'hui sur un terreau favorable : l'évolution de la nature des conflits, de moins en moins interétatiques ; la désagrégation de certains États et la « milicianisation » (Marchal 1997 : 33) croissante des sociétés africaines; la réduction générale des forces armées consécutive à la fin de la guerre froide, qui met sur le marché surabondance d'hommes et de matériel ; les contraintes budgétaires des gouvernements occidentaux forcés de restructurer leur appareil militaire ; leur répugnance croissante à intervenir dans les «zones grises » depuis l'échec de l'opération américaine en Somalie ; la faveur dont jouit la doctrine « zéro mort »; les résultats mitigés des actions menées par les Casques Bleus ainsi que la diffusion planétaire du credo libéral, sont autant de facteurs qui se conjuguent pour faire de la « sécurité privée » un marché en pleine expansion.

Dominé par les firmes britanniques (DSL notamment), américaines (MPRI, Wackenhut), sud-africaines (Executive Outcomes) et israéliennes (Levdan), ce secteur compte désormais des dizaines, voire des centaines de sociétés, allant du simple gardiennage à la guerre « clés en main ». Cette nébuleuse mercenaire commence à être assez bien documentée ${ }^{2}$ mais nous nous limiterons ici aux cas paradigmatiques de la société britannique Sandline International et de la célèbre firme sud-africaine Executive Outcomes (EO).

Distinctes, ces deux entreprises entretiennent des liens étroits, chacune servant à l'occasion de sous-traitant à l'autre. Toutes deux sont des filiales d'une vaste holding - Strategic Resources Corporation (SRC) - fondée par un homme devenu 
en quelques années le symbole du « renouveau mercenaire »: Eeben Barlow. Ex-commandant en second du fameux 32e bataillon Buffalo des Forces armées sud-africaines (SADF) qui opérait en Angola, Barlow fut surtout un membre actif du redoutable Civil Cooperation Bureau (CCB), responsable de l'assassinat de nombreux militants anti-apartheid. Anticipant les inévitables reconversions liées au démantèlement des « forces spéciales » anti-apartheid, il fonda Executive Outcomes en 1989. Bien qu'innovante, cette initiative s'inscrivait dans la continuité de la «stratégie totale », lancée dès la fin des années soixante-dix par Pretoria, qui consistait à privatiser les unités secrètes des SADF.

Sandline International, quant à elle, est dirigée par le lieutenant-colonel Tim Spicer, ancien officier de l'armée britannique et héros de la guerre des Malouines. Formé à la lutte antiterroriste en Irlande du Nord, il a exercé des responsabilités de conseiller pendant la guerre du Golfe et a fait partie de l'équipe du général Rose en Bosnie avant de créer sa société de sécurité avec les Sud-Africains d'Executive Outcomes et d'autres soldats de Sa Majesté reconvertis dans les affaires.

Ces nouveaux mercenaires n'ont plus grand chose à voir avec les « affreux » du Katanga et les baroudeurs du temps de Bob Denard ou de Mike « le fou ». Ce sont de véritables entrepreneurs militaires, qui ne se limitent pas au créneau de la guerre et de la sécurité. Jouant à merveille des opportunités de la globalisation, ils s'appuient sur une vaste organisation visant à l'intégration verticale des activités de « mise en valeur » et d'exploitation des rentes du continent africain, en particulier des ressources minières et pétrolières. Basée à Pretoria et à Londres, la holding SRC comprend une vingtaine de sociétés, pour la plupart domiciliées dans des paradis fiscaux ou des places offshore (l'adresse principale de Sandline, par exemple, est aux Bahamas), qui occupent chacune une niche lucrative : sécurité (Executive Outcomes, Sandline, Lifeguard), exploitation de mines d'or et de diamants (Branch Energy, Diamond Works), exploitation pétrolière et gazière (Heritage Oil and Gas), transport aérien (Ibis Air), déminage, travaux de réhabilitation, infrastructures médicales, informatique, services financiers... À Londres, ces sociétés sont rassemblées dans le groupe Plaza 107 où se côtoient notamment Tim Spicer et Tony Buckingham, ancien officier des SAS devenu PDG d'Heritage Oil. Disposant d'un vaste réseau de relations politiques à travers le monde, ce dernier joue un rôle pivot dans la holding dont il paraît être l'éminence grise.

Sandline, comme sa consœur Executive Outcomes, propose une large gamme de services allant du conseil aux opérations militaires de grande envergure et couvrant un vaste champ (création d'unités de renseignement, déminage, protection

1. Cité par Thomas J. Wertenbaker, «Virginia under the Stuarts, 1607-1688 », in The Shaping of Colonial Virginia, New York, Russell and Russell, 1914, pp. 29-30.

2. Voir bibliographie. Les informations signalées par un astérisque ont été recueillies lors d'entretiens. 
rapprochée, surveillance maritime, lutte contre le trafic de drogue...) qui s'étend à la sphère commerciale. Dans ce domaine, Tim Spicer se targue de fournir des « services plus sophistiqués $»^{*}$ que le simple gardiennage d'entreprises ou de plateforme pétrolière : évaluation des risques-pays, renseignement économique, infrastructure médicale, surveillance aérienne... Spicer affirme également s'intéresser de près aux questions de collecte des taxes, en particulier à l'administration des douanes où il envisage de s'investir à la manière des entreprises qui opèrent déjà en Afrique centrale et occidentale (SGS, Veritas). Sachant que la plupart des sociétés du groupe SRC sont domiciliées dans des paradis fiscaux, on peut émettre l'hypothèse que ces techniques «plus sophistiquées » de sécurité économique comprennent également des conseils en matière de placements financiers offshore, de fraude fiscale, voire de blanchiment d'argent. Les services proposés par le site internet du groupe SRC - basé dans un pays d'Amérique latine - nourrissent cette hypothèse : « tax free income; offshore confidential banking; free from exchange controls; offshore registered agent and registered office ; offshore escrow, save cash from seizure! »

Tim Spicer souligne aussi le vif intérêt porté par sa firme aux affaires humanitaires, qui constituent un « domaine prometteur $»^{*}$ : escorte de convois, sécurisation des zones, soutiens et formation en matière médicale... Le secteur humanitaire présente à ses yeux le triple avantage d'être en pleine expansion, d'assurer une bonne image internationale et d'établir des liens étroits avec les agences de l'ONU. Il semble d'ailleurs que les rapports entre les ONG d'urgence et les sociétés de sécurité tendent à se banaliser depuis que les premiers contacts ont été établis en Somalie et au Libéria. Dans le même ordre d'idée, Sandline envisage d'investir dans les programmes de réhabilitation d'infrastructures, sanitaires ou autres.

\section{La réinvention de l'économie concessionnaire}

L'organigramme et le fonctionnement de la holding SRC mettent en évidence la synergie établie entre l'activité guerrière et l'exploitation minière des pays « libérés », c'est-à-dire entre les sociétés de sécurité au sens strict et les sociétés commerciales du groupe Branch-Heritage dirigé par Tony Buckingham. Il semble bien que les membres du réseau Barlow-Spicer-Buckingham monnayent leur assistance militaire contre des concessions minières ou des permis d'exploitation d'autres ressources naturelles, qui donnent lieu à des contrats en bonne et due forme. C'est ce que Chapleau et Misser appellent « security equity swaps », par analogie avec la technique des debt equity swaps, qui consiste à convertir des créances en actifs de sociétés appartenant aux pays débiteurs. Il va sans dire que les gouvernements africains qui font appel à des services mercenaires ne sont généralement pas dans les conditions optimales pour négocier. En Sierra Leone, les rebelles étaient à vingt kilomètres de la capitale quand les troupes d'EO sont intervenues en mai 1995. 
Ayant rapidement sécurisé Freetown, les mercenaires ont ensuite pris le contrôle des mines diamantifères de Kono puis des gisements de Sierra Rutile, avant de lancer l'assaut contre le QG de la rébellion en octobre 1996. Le gouvernement de V. Strasser, incapable de payer une facture qui s'élevait à un million de dollars par mois, accorda à Branch Energy, filiale de la holding SRC, puis à Diamond Works, dont est actionnaire Tim Spicer, l'exploitation des mines de diamant dans les zones conquises par les troupes d'EO.

En Angola, Branch Energy et Diamond Works ont obtenu l'exploitation de cinq immenses concessions diamantifères. Arrivés à Luanda en 1993 avec un contrat de 40 millions de dollars, les mercenaires d'EO avaient aidé le gouvernement Dos Santos à moderniser ses forces pour repousser la menace de l'UNITA (qui bénéficiait auparavant de leur appui). Dès juin 1994, ils avaient délogé les rebelles des installations pétrolières de Soyo et pris le contrôle des zones minières de Cafunfo. Sous la pression du FMI et des États-Unis, EO dut toutefois quitter le pays à la fin de 1995 et fut remplacée par la compagnie américaine MPRI, mais la holding d'Eeben Barlow parvint à maintenir ses acquis dans le secteur minier par le biais de filiales.

En Ouganda, l'usage de la coercition privée est moins apparent tant les Uganda People's Defence Forces (UPDF) occupent le terrain militaire et politique. Pourtant, il est avéré que les services d'EO ont été utilisés par le gouvernement dans sa lutte contre la rébellion nordiste de la Lord Resistance Army, et que d'étroites connexions existent entre la holding mercenaire et le pouvoir ougandais. Caleb Akwandanaho (alias Salim Saleh), le « demi-frère » du président Museveni et son conseiller spécial pour les affaires de défense, en charge de la «pacification » du Nord, est en effet le représentant en Ouganda de certaines filiales de la SRC (Branch Energy pour l'exploitation minière, Saracen pour la sécurité), dans lesquelles il détient des parts importantes. Sous sa houlette, Saracen-Uganda diversifie depuis 1997 ses activités dans le secteur électronique et dans la vente d'armes de poing. Le groupe Branch-Heritage s'est aussi vu confier des concessions aurifères dans le parc national de Kidepo et l'exploitation d'un gisement pétrolier au sud du Lac Albert.

Chapleau et Misser remarquent que la nébuleuse d'EO n'est pas la seule à combiner ainsi services mercenaires et concessions minières. En partenariat avec la compagnie American Fields Mineral de Jean-Raymond Boulle, la société belgonéerlandaise International Defense and Security (IDAS), observent-ils, a obtenu en Angola une concession diamantifère plus vaste que la Belgique en échange de ses services en matière de sécurité. Au Congo-Brazzaville, la firme israélienne Levdan s'est vu accorder la moitié des parts du permis pétrolier d'exploitation Marine III par le gouvernement Lissouba, dont les milices avaient été formées par des spécialistes israéliens : une pratique courante au Congo, où les dettes gagées sur le pétrole et les paiements « en nature » sont devenus un des principaux moyens de financement de l'État. 
Faut-il voir dans ces pratiques militaro-commerciales un retour à l'économie concessionnaire, voire une réinvention de «l'Afrique des comptoirs » (Mbembe 1992 ; Bayart et al. 1997) ? Les analogies sont effectivement frappantes entre ces techniques modernes de prédation minière menées par les sociétés de sécurité et l'activité des grandes compagnies concessionnaires qui, avant et pendant la colonisation, assurèrent leur prospérité par la militarisation du commerce, en se faisant octroyer, au besoin par la coercition, des contrats exclusifs d'exploitation des ressources naturelles (Coquery-Vidrovitch 1972). À l'instar des nouvelles holdings mercenaires, les grandes maisons de commerce de l'époque disposaient de l'usage de la « violence légitime » en lieu et place des États, assumaient des fonctions fiscales de collecte des taxes et investissaient dans la construction d'infrastructures. « Ces compagnies, écrit Janice Thomson, n'étaient pas simplement autorisées à employer la violence, mais étaient aussi dotées de presque tous les pouvoirs de souveraineté » (Thomson 1994 : 32), lesquels étaient volontairement délégués par les États. Dans les colonies britanniques, mais aussi françaises et portugaises, l'indirect rule ne s'appuyait pas seulement sur les structures de la chefferie coutumière mais aussi et surtout sur des sociétés privées, quasi omnipotentes sur le territoire.

Cette comparaison avec l'économie concessionnaire a le mérite de situer les collusions des gouvernements africains avec les sociétés de sécurité dans la continuité historique des modes de gestion de l'extraversion indissociables du processus de formation de l'État en Afrique (Bayart 1989). Mais elle ne doit pas faire oublier que les mercenaires entrepreneurs agissent désormais dans un contexte de globalisation financière qui leur ouvre de nouvelles opportunités d'accumulation. Elle ne doit pas non plus conduire à interpréter ce phénomène comme une « recolonisation déguisée » et privatisée : si les termes de l'échange sont à l'avantage des mercenaires, les « États faibles » tirent aussi de ces arrangements militaro-commerciaux matière à consolidation ; en outre, ces « recolonisés » conservent souvent une large marge d'autonomie, en raison de la concurrence qui existe dans le secteur. L'exemple ougandais de Salim Saleh, qui a créé sa propre société multiservices, Calebs International, mais aussi la floraison des sociétés de sécurité en Angola, au Cameroun et en Afrique du Sud, témoignent de ce que ces joint ventures peuvent conduire à un véritable entreprenariat militaro-commercial africain.

\section{Légalité et moralité dans la guerre : les faux-semblants de la vertu mercenaire}

Pourtant, contre toute évidence, les responsables de Sandline tiennent absolument à dissocier les affaires minières et guerrières de la nébuleuse dont l'articulation est présentée comme fortuite (« Nous partageons le même immeuble et la même société de services, Plaza $107 \gg^{*}$ ). Craignant d'être accusé de faire la guerre pour 
prendre le contrôle des ressources minières, notamment d'or et de diamant, Tim Spicer concentre son argumentation sur le secteur de la sécurité et revendique sa légitimité au sein de celui-ci en présentant ses services dans un discours bien en phase avec l'idéologie du moment.

L'activité essentielle de Sandline International comme d'EO demeure, selon lui, l'action militaire et le soutien aux armées nationales. Dans ce domaine, l'accent est mis sur l'entraînement des troupes - présenté comme une opération de transfert de technologie - et sur le conseil en matière stratégique. Comme le scandale de Sierra Leone l'a prouvé, ces appuis peuvent s'accompagner de services en matière d'équipement ${ }^{3}$, mais Sandline comme Executive Outcomes se défendent d'être des marchands d'armes. Soucieuses de respectabilité, elles cherchent de toute évidence à se débarrasser de leur image de « chiens de guerre » et affichent leur désir de collaborer avec les instances multilatérales et les gouvernements occidentaux à la faveur de la réticence de plus en plus grande des unes et des autres à intervenir dans les zones de conflit. À cette fin, elles déploient des stratégies de communication élaborées ${ }^{4}$. Elles se glissent sans peine dans le moule néo-libéral de la Banque mondiale et inscrivent leur action dans une tendance généralisée à la privatisation de services autrefois assurés par l'État. Enfin, pour rompre avec l'image des « affreux », elles mettent en avant leur détermination à se conformer à un code moral énoncé sous forme de « principes opérationnels », dont le premier est «l'assurance de confidentialité pour le client $»^{5}$ :

${ }_{n}$ Un $\ll$ recrutement aux standards internationaux $»^{*}$ : si les officiers et le personnel d'encadrement d'EO et Sandline sont généralement issus des unités d'élite sud-africaines et britanniques, plus de $60 \%$ des soldats engagés par les deux sociétés sont africains, principalement originaires d'Angola et du Mozambique. En recrutant prioritairement parmi les anciens « Buffalo », « Reccies », « Parabats » et autres « Crowbar $»^{6}$, les deux sociétés se sont constitué un réseau de milliers de soldats de fortune expérimentés et formés aux opérations spéciales en Afrique australe. Plutôt que de véritables armées privées, il s'agit donc plutôt de réseaux informels de combattants et de techniciens rapidement mobilisables.

\footnotetext{
3. Léquipement high-tech ne semble pas avoir les faveurs du groupe. EO et Sandline utilisent certes un matériel perfectionné (hélicoptères Mi-8, Mi-17 et Mi-24; chasseurs Mig 23 et 27 ; systèmes à infra-rouge etc.) mais la simplicité et la fiabilité d'un matériel « adapté au contexte africain » ont leur préférence. Tim Spicer considère que les gadgets utilisés par la firme américaine MPRI ne sont que «bullshit ».

4. Presque toutes ces sociétés ont leur site web qui sert à présenter leurs activités, mais aussi à recruter des « soldats de fortune $\gg$ (voir bibliographie).

5. Document de présentation de Sandline International.

6. Désignant respectivement les membres du 32e bataillon dirigé par E. Barlow et J. Breytenbach (le propre frère du poète anti-apartheid Breyten Breytenbach !), des Commandos de reconnaissance, de la Brigade parachutiste et de la milice paramilitaire Koevoet, spécialisée dans la contre-insurrection.
} 
$\mathrm{n}$ « Aucune activité de nature illégale »* telle que le trafic de drogue, de matières fissiles, l'utilisation du terrorisme... Affirmé avec force, ce principe n'empêche pas le lieutenant-colonel Spicer de reconnaître qu'il ne se préoccupe absolument pas de la provenance des fonds mobilisés par ses clients pour le payer. Le fait que l'argent puisse venir du trafic de drogue «would be a concern », mais globalement il considère que cela n'est pas son problème*.

n Tim Spicer met surtout en avant le fait que sa société, tout comme EO, ne travaille « qu'avec des gouvernements légitimes, reconnus internationalement et - de préférence - élus démocratiquement $\gg^{*}$. Sandline International, comme ses consœurs, affirme refuser de traiter avec les pays sous embargo ou contrôle international tels le Soudan, l'Irak ou la Corée du Nord, et pose comme principe de ne pas aider les mouvements révolutionnaires ni les insurrections armées, pour mieux se démarquer du mercenariat des années soixante et soixante-dix. On peut s'interroger sur les critères de « légitimité » retenus par les marchands de sécurité dans l'établissement de leurs contrats, et considérer que « c'est de cet argument ambigu - le fossé entre légalité et légitimité ne cessant de se creuser dans un certain nombre de pays - qu'[ils] tirent la marge de manœuvre nécessaire à la conduite de leurs affaires » (G. de Saint-Quentin 1998 : 38). Mais, en dépit de ses ambivalences et de son hypocrisie, cette approche « légitimiste » révèle deux aspects importants pour saisir les dynamiques actuelles de privatisation de la guerre en Afrique et comprendre l'innovation que constitue l'émergence de ces nouveaux acteurs sur la scène stratégique internationale.

En premier lieu, on constate par ce biais que la privatisation de la coercition en Afrique ne traduit pas forcément un contournement de l'autorité de l'État, mais s'effectue, bien au contraire, avec le concours actif de celui-ci, en empruntant toutes les formes de la légalité institutionnelle. Autrement dit, la perte du monopole étatique de la violence et la déterritorialisation de celle-ci ne signifient pas obligatoirement l'effondrement des États ni la victoire des « Empires transnationaux » sur le «monde statocentré ».

Dans ses travaux sur la Sierra Leone et le Libéria, W. Reno a bien montré que les collusions qui s'établissent entre les dirigeants de ces multinationales de la sécurité et les gouvernements-clients contribuaient à la reproduction des réseaux de patronage de l'« État-rhizome » (Bayart 1989), et finalement à la «stabilisation » de celui-ci, fût-ce sur le mode prédateur. Et, si l'on suit le raisonnement de Béatrice Hibou dans ce même numéro, il s'agirait moins d'une « démission » des États face aux condottieres que d'une « autre manière d'affirmer leur souveraineté et leur pouvoir de contrôle dans un contexte de dépendance accrue et de crise économique grave » : une forme de gouvernement indirect par délégation de la force militaire à des compagnies déterritorialisées qui fut d'ailleurs longtemps la norme, comme le montre Janice Thomson (1994). 


\section{Les tentations de la « décharge »}

Cette volonté de respecter une façade de légalité internationale témoigne du désir des sociétés de sécurité de voir leur action « reconnue d'utilité publique » par la communauté internationale. Le dernier « principe opérationnel » avancé par Sandline en témoigne : «Rien n'est entrepris qui aille contre les intérêts nationaux des gouvernements occidentaux ${ }^{*}$. Conscients que la survie de leur entreprise en dépend largement, les nouveaux condottieres assurent qu'ils « soutiennent la politique extérieure des pays occidentaux $»^{*}$ et que le développement de la sécurité privée coïncide avec les objectifs des grandes puissances soucieuses de se désengager militairement du continent africain. De fait, ils se disent prêts à monter des joint ventures avec eux dans le domaine de la coopération militaire Nord-Sud.

Le marketing mercenaire adopte alors le langage en vigueur dans les instances bi- et multilatérales de coopération. Sandline et EO, selon Tim Spicer, favorisent « le développement par le commerce » en assurant la stabilité du continent et en garantissant la sécurité des investissements. Elles s'inscrivent même dans une problématique de la «bonne gouvernance », en prétendant contribuer à la restauration de l'autorité de l'État (fût-ce sur le mode de la privatisation de ses fonctions régaliennes) et de « la qualité de la vie » (sic !). Les nouveaux condottieres ont compris qu'il leur fallait investir dans le créneau du maintien de la paix, tant pour acquérir une respectabilité sur la scène stratégique mondiale que pour assurer la diversification et la pérennisation de leurs activités. «We are peace enforcers », proclament-ils, espérant être confirmés dans ce rôle par une communauté internationale impuissante à assurer l'ordre et la sécurité. Se considérant comme l'avant-garde des Casques Bleus, ils posent franchement la question de leur mode de rétribution : «Pourquoi ne pourrions-nous pas être payés par la communauté internationale, par un pays tiers, voire par le FMI ? »**

Signe de la levée d'un tabou, cette question est d'ailleurs évoquée sérieusement par des intellectuels néo-libéraux influents tels que les époux Toffler : «Quand les nations ont déjà perdu le monopole de la violence, pourquoi ne pas envisager de créer des forces de mercenaires volontaires, organisées par des entreprises pour mener des guerres sur une base contractuelle pour le compte des Nations unies ? $\gg 7$. Si de tels contrats n'ont pas été formellement établis entre l'ONU et les compagnies de sécurité, les services d'EO en Sierra Leone n'en ont pas moins été en partie payés grâce aux fonds déboursés par le FMI au titre de l'ajustement structurel. Près de la moitié des crédits auraient ainsi été versés aux mercenaires avec l'assentiment des institutions de Bretton Woods, dans ce qui s'apparente à une 
opération de « blanchiment » d'argent (à rebours) par le canal des finances publiques d'un « gouvernement légitime ». Un montage similaire a été consenti par la Banque mondiale en Angola pour les mêmes raisons ${ }^{8}$.

Il semble d'ailleurs que des rapports directs aient d'ores et déjà été établis entre les sociétés de sécurité et les agences multilatérales. Selon Tim Spicer, des discussions informelles auraient eu lieu avec des représentants des institutions de Bretton Woods qui seraient « en voie d'adopter une attitude plus positive $»^{*}$ au vu des « succès » obtenus par EO en Angola et en Sierra Leone. Il affirme aussi avoir pris langue avec le Secrétaire général de l'ONU, qu'il aurait connu lors de sa mission pour l'IFOR en Bosnie. Contacté lors de la crise burundaise, Kofi Annan l'aurait invité à lui présenter des propositions, puis se serait rétracté. En vérité, ce dernier ne paraît pas très disposé à collaborer avec des sociétés qu'il continue de qualifier de mercenaires. Mais, sur le terrain, les contacts sont fréquents : en juillet 1998, la presse sud-africaine révélait par exemple que Lifeguard, une des sociétés d'EO, travaillait directement pour l'ONU en Sierra Leone, fournissant essentiellement des services de gardiennage. Et, selon une analyse des services de renseignement britanniques, il se pourrait également qu'à moyen terme l'OUA « se voie forcée d'offrir à Executive Outcomes un contrat pour la gestion du peacekeeping sur l'ensemble du continent $\gg^{9}$.

Sur le plan bilatéral, il est clair que la collaboration avec les grandes puissances est plus avancée. Le scandale des trafics d'armes avec la Sierra Leone évoqué au début de cet article n'a été que le révélateur de collusions qui existent depuis assez longtemps et qui tendent à se multiplier. La plupart des sociétés britanniques de sécurité ont en effet été créées et sont dirigées par d'anciens officiers des Special Air Services (SAS) qui ont gardé d'étroits contacts avec les états-majors et les services de renseignement : outre Sandline, l'autre grande compagnie, Defence Systems Ltd (DSL), a été fondée en 1981 par Alistair Morrison, ex-commandant en second du $22^{\mathrm{e}}$ régiment $\mathrm{SAS}$, et compte dans son conseil d'administration d'illustres officiers dont le vicomte Gilbert Monckton de Brenchley, détenteur de l'ordre de l'Empire britannique. Dans les années quatre-vingt, Keenie Meenie Services (KMS) et Saladin Security partageaient des bureaux situés à proximité des quartiers généraux du 22e régiment SAS.

Les passerelles sont encore plus visibles aux États-Unis. Depuis les années cinquante, Wackenhut collabore avec la CIA dont un des célèbres patrons, Bill Casey, avait été auparavant avocat de la compagnie. Créée en 1987 par de hauts gradés à la retraite, Military Professional Resources Inc. (MPRI) se targue, dans ses plaquettes publicitaires, d'être sise à côté du Pentagone et de compter dans son fichier des milliers de noms d'officiers de l'armée américaine. Cette société, aujourd'hui principale concurrente d'EO, est effectivement dirigée par d'anciens militaires ayant exercé de hautes responsabilités et compte dans son conseil d'administration des hommes 
politiques qui furent, à la Maison Blanche, en charge de dossiers sensibles. Une autre de ces sociétés, BDM International, est contrôlée par le groupe Carlyle dirigé par James Baker, ancien Secrétaire d'État, Richard Darman, ex-directeur du Budget à la Maison Blanche et Frank Carlucci, ex-secrétaire d'État à la Défense. La compagnie SAIC, quant à elle, compte dans ses rangs deux anciens ministres de la Défense (William Perry et Melvin Laird) et deux anciens directeurs de la CIA (Robert Gates et John Deutch). De fait, une véritable osmose existe entre le Pentagone et ces multinationales sécuritaires auxquelles il sous-traite de plus en plus fréquemment les opérations délicates : ce fut, semble-t-il, le cas en ex-Yougoslavie, où le MPRI a participé à la modernisation de l'armée croate et surtout à l'opération d'août 1995 en Krajina, avec l'aval du gouvernement américain. Ce fut également le cas de la petite firme Ronco, composée d'anciens des Special Forces, qui, sous couvert d'un contrat de déminage au Rwanda, a vraisemblablement participé à l'offensive contre Mobutu en entraînant les troupes rwandaises sur la base de Cyangugu, avec l'assentiment du National Security Council et de l'attaché militaire américain à Kigali, Tom Odum. Les responsables américains du renseignement militaire, qui apprécient l'avantage de se décharger ainsi de leurs interventions sur le secteur privé, tentent de rationaliser et d'institutionnaliser cette collaboration : depuis 1997, des réunions confidentielles sont organisées tous les six mois par la Defense Intelligence Agency avec les dirigeants des compagnies privées américaines et sud-africaines, sous la houlette du cabinet Cohen \& Woods créé par William Cohen, secrétaire d'État aux Affaires africaines sous la présidence Bush. De fait, l'action de ces entreprises coïncide souvent avec les intérêts de leur État d'origine.

\section{Les risques de la sous-traitance}

«Du fait de la nature de nos affaires, constate Tim Spicer, nous devons maintenir de bonnes relations avec les pays occidentaux. Non pas des relations formelles, mais des rapports d'intérêt », ajoutant qu' « il serait contre-productif pour les deux parties de ne pas communiquer $»^{*}$. Le renforcement des contacts entre $\ll$ les deux parties » que l'on observe actuellement aux États-Unis, en Grande-Bretagne, mais aussi en France indique à tout le moins que les dirigeants occidentaux considèrent que cette « communication » doit bel et bien être établie, voire, selon D. Shearer (1998: 76), que « le temps est venu de mener une politique d'engagement constructif avec les compagnies militaires $\gg$.

\footnotetext{
8. « La plus grande partie des recettes de l'État en 1995 est venue de prêts consentis par le FMI, de certaines aides et de revenus douaniers. Le FMI avait donné son accord pour que le paiement de la facture d'EO fasse partie du budget de la Sierra Leone, considérant que la stabilité est une nécessité pour attirer les investissements » (Shearer $1998: 53$ ).

9. Document cité par le Weekly Mail, 9 février 1997.
} 
On peut concevoir qu'il soit important de « dialoguer » avec des acteurs qui ont d'ores et déjà acquis un poids économique et stratégique non négligeable et qui pourraient, à moyen terme, acquérir une réelle influence politico-militaire sur le continent africain ${ }^{10}$. Les occasions d'intervention se multipliant, il est à craindre en effet que le pouvoir des sociétés telles qu'EO ne se consolide à l'avenir, dans la mesure où la stratégie d'accumulation poursuivie - sécurisation, puis exploitation minière - assure à ces holdings mercenaires des ressources importantes et durables.

On peut également imaginer que, au moment où la France réduit son implication au sud du Sahara, la tentation soit grande de déléguer la gestion des crises africaines à des sous-traitants privés qui présentent le profil soigneusement travaillé de peace enforcers. Ceux-ci ont compris tout le parti qu'ils pouvaient tirer de la conjoncture et tentent d'établir des relations plus étroites avec les autorités françaises. Des projets de collaboration sont évoqués à demi-mot, notamment dans le domaine de la formation et de l'entraînement des troupes. Et il n'est pas impossible qu'au ministère de la Défense, au Quai d'Orsay ou dans les milieux du renseignement, ces projets trouvent une oreille de plus en plus attentive dans une phase où la coopération militaire française va devoir s'adapter à la mise en place de structures régionales de sécurité en Afrique (projets RECAMP et ACRI). Officiellement, la France entend maintenir son appui militaire classique dans le cadre des futures forces d'intervention régionales, comme l'ont prouvé, début 1998, les grandes manœuvres « Guidimaka » au Sénégal. Mais, sous couvert d'« africanisation » et de «multilatéralisation » de la gestion des crises au sud du Sahara, on peut imaginer que les opérations les plus délicates soient un jour confiées, de manière contractuelle, à ces nouveaux « professionnels ${ }^{11}$. En bref, dans un contexte de restrictions budgétaires et de réforme de la politique africaine de la France, il peut être tentant de se parer du manteau de la vertu multilatérale tout en perpétuant l'ancienne politique des réseaux militaires par le biais d'intermédiaires privés ${ }^{12}$.

Il convient toutefois de mesurer les risques que présente le passage de la privatisation de la guerre à celle du peacekeeping, voire à celle de la coopération bi- et multi-

\footnotetext{
10. L'Afrique est clairement désignée par T. Spicer comme la zone privilégiée d'intervention des troupes mercenaires, du fait, d'abord, de l'instabilité qui y règne, mais aussi et surtout en raison des possibilités d'obtenir des résultats rapides et spectaculaires avec un minimum d'investissement en hommes et en matériel. Selon Spicer, ce rapport est moins favorable au Proche et au Moyen-Orient ou dans les pays de l'ex-bloc soviétique : du fait du niveau d'armement des parties en conflit, le « retour sur investissement $\gg y$ est moins rapide, en quelque sorte.

11. C'est à peu près la configuration qui semble avoir prévalu en Sierra Leone, où Executive Outcomes a bénéficié de l'appui aérien et de l'artillerie des forces «multilatérales » de l'ECOMOG (en vérité nigérianes).

12. On retrouverait ainsi, dans le domaine de la coopération militaire, la politique du double jeu qui prévaut depuis 1994 dans le domaine de l'aide financière, où la « doctrine d'Abidjan », qui devait imposer une nouvelle conditionnalité multilatéralisée, a subi nombre d'entorses, traduisant le souci de Paris de conserver des liens privilégiés avec les pays amis du pré-carré.
} 
latérale. Certes, les sociétés privées peuvent se prévaloir de certains « succès » en Croatie, en Angola ou en Sierra Leone, et disposent d'incontestables atouts. La souplesse de leur organisation leur permet de s'adapter rapidement à l'évolution des situations conflictuelles et de répondre précisément aux besoins de leurs clients en leur proposant des solutions à la carte (après des « études de faisabilité » facturées en millions de dollars). Étant donné leurs caractéristiques opérationnelles et leurs dispositifs tactiques, les nouvelles compagnies de mercenaires sont aussi mieux à même d'affronter des situations de guerre civile que les armées occidentales tenues d'intervenir en Afrique dans le cadre d'accords de défense obsolètes. Elles sont donc bien en phase avec l'évolution de la conflictualité depuis la fin de la guerre froide.

Mais leur « tableau de chasse » ne doit pas faire illusion. D'abord Sandline ou Executive Outcomes sont loin d'avoir prouvé leur capacité de peace enforcers. Si elles peuvent renverser des situations militaires compromises et aider les gouvernements à signer des accords de paix dans des termes plus favorables (par exemple les accords de Lusaka en 1994), elles ne semblent pas en mesure d'assurer la stabilité des pays sur le moyen terme : la reprise de la guerre en Sierra Leone et en Angola est là pour le prouver. Ces sociétés n'ont pas vocation à assurer le « suivi » de leur action : «L'objectif n'est pas de s'implanter dans les pays-clients, précise Tim Spicer : dès que le contrat est terminé, on s'en va $»^{*}$. Ensuite, en renforçant la militarisation des pays, en accélérant le « transfert de technologie » et la diffusion d'armements de plus en plus sophistiqués, les sociétés de sécurité accroissent le potentiel de violence. D'autant plus que les ex-officiers sud-africains appliquent les stratégies pratiquées du temps de l'apartheid en formant des mouvements du type Inkatha. La transformation des chasseurs « traditionnels » Kamajor en une véritable faction armée, disposant de matériel moderne, en Sierra Leone, en est le meilleur exemple. L'arrivée de mercenaires a souvent pour conséquence de radicaliser les parties en conflit, et notamment de nourrir le registre nationaliste. Elle conforte le choix des solutions militaires au détriment du règlement politique des conflits. Elle peut aussi avoir pour conséquence d'intensifier les luttes factionnelles au sein des «États faibles » et d'accentuer la tendance au warlordism. C'est le cas en Angola où, depuis l'intervention d'EO, le nombre de sociétés de ce type s'est multiplié de manière vertigineuse, contribuant à l'exacerbation des rivalités politico-militaires. Ce fut aussi le cas en Papouasie-Nouvelle-Guinée, où l'arrivée de Sandline a provoqué une déflagration politique qui s'est terminée par une tentative de coup d'État du général Singirok.

En quatrième lieu, on peut émettre des doutes quant à la volonté des mercenaires de résoudre des conflits qui sont leur gagne-pain, tout comme il est vain de penser que la logique d'accumulation économique décrite plus haut les poussera à rechercher la stabilité. Certes, les compagnies comme EO, Sandline ou MPRI ont compris qu'il leur fallait exploiter le créneau du « maintien de la paix » pour 
acquérir une légitimité internationale et diversifier leurs activités. Mais l'« économie de pillage » qu'elles ont mise en place dans l'exploitation des rentes du sous-sol africain s'accorde bien mieux avec l'incertitude, la violence et la criminalisation du pouvoir qu'avec la pacification. À plus ou moins long terme, on peut d'ailleurs craindre que la rivalité commerciale croissante de ces entrepreneurs-militaires ne se traduise par des affrontements entre armées privées, éventuellement sous le couvert d'États « protecteurs ${ }^{13}$. Les indices d'une telle évolution existent déjà, comme en témoignent les tensions croissantes entre EO et MPRI, le rôle joué par la firme J\&S Franklin dans l'échec de Sandline en Papouasie ou encore les projets concurrents d'Eeben Barlow et de Sean Cleary, patron la compagnie Strategic Concepts, en Angola.

Enfin, il faut souligner le caractère incontrôlable de ces sociétés, qui ont d'ores et déjà monté de véritables armées de métier au matériel sophistiqué. «Affranchies des contraintes territoriales, elles ont parfaitement pris en compte les failles juridiques consécutives à la mondialisation de l'économie et élaboré des réseaux de financement et de sociétés écrans sur lesquels aucune loi n'a prise », écrit Grégoire de Saint-Quentin (1998 : 39). Une de leurs caractéristiques majeures est que leur configuration juridique et leur raison sociale évoluent constamment. La dynamique de scissiparité - multiplication à l'infini des sociétés écrans, délocalisation des activités dans le monde entier... - qui sous-tend, par exemple, le meccano de la holding d'Eeben Barlow montre que les pratiques d'accumulation des nouveaux « chiens de guerre » répondent bien plus aux logiques de dédoublement de «l'Afrique invisible » (Bayart 1995-96) qu'aux critères de transparence de la good governance.

Les bienfaits de la « marchandisation » de la sécurité chantés par certains néo-libéraux nous paraissent donc une chimère inquiétante. Or, étant donné la montée en puissance des nouveaux mercenaires et les hésitations croissantes des grandes puissances à intervenir directement, on peut supposer que la tendance à la privatisation du peace enforcing va s'accentuer dans les années à venir. Pour autant, cette dynamique ne signifie pas contournement des États par les multinationales de la sécurité comme le voudrait la vulgate transnationaliste : il s'agit bien plutôt d'une diplomatie de gestion indirecte des risques, d'une délégation de la coercition, engagée par des autorités publiques « qui en gardent le contrôle direct, ne serait-ce que par l'intermédiaire du "pantouflage" et du "chevauchement" permanent entre le privé et le public $\gg$ (Bayart 1998).

13. Une configuration à rapprocher de celle qui prévalait, par exemple, entre les grandes Compagnies commerciales du XVII ${ }^{\mathrm{e}}$ au XIXe siècle et que décrit Janice Thomson. 
Comme James Ier avec la Compagnie de Virginie, les grandes puissances espèrent ainsi combiner un maximum de liberté et un minimum de responsabilité. Mais, en cédant à la tentation, elles se placent devant le même dilemme que les chefs d'État du XVII ${ }^{\mathrm{e}}$ siècle face aux corsaires et aux grandes compagnies de commerce (Thomson 1994) : comment contrôler ces agents tout en leur laissant suffisamment de liberté pour garantir leur efficacité et les inciter à prendre les risques du commerce de la guerre ? Surtout, elles devraient se rappeler que les monarchies de l'époque se sont trouvées entraînées au bord de la guerre généralisée pour avoir trop cru aux avantages de la sous-traitance ${ }^{14}$.

14. Je remercie Stephen Ellis pour sa lecture attentive de la première version de cet article.

\section{Bibliographie}

BAYART (Jean-François), 1989. L'État en Afrique. La politique du ventre, Paris, Fayard.

BAYART (Jean-François), 1995-96. "L'Afrique invisible ", Politique internationale, n70, hiver, pp. 287-295.

BAYART (Jean-François), 1998. " Même la guerre se privatise ", Croissance, n416, juin, p. 50.

BAYART (Jean-François), Eluıs (Stephen), Hibou (Béatrice), 1997. La Criminalisation de l'État en Afrique, Bruxelles, Complexe.

CoquerY-VIDrovitch (Catherine), 1972. Le Congo au temps des grandes compagnies concessionnaires (1898-1930), Paris, Mouton-EHESS.

EluIs (Stephen), 1998. " The historical significance of South Africa's Third Force ", Journal of Southern African Studies 24(2), juin, pp. 261-299.

HiBou (Béatrice), 1998. "Retrait ou redéploiement de l'État ? ", Critique internationale, $\mathrm{n}^{\circ} 1$, automne, pp. 151-168.

Marchal (Roland) et Messiant (Christine), 1997. Les Chemins de la guerre et de la paix. Fins de conflit en Afrique orientale et australe, Paris, Karthala.

Mbembe (Achille), 1992. " Afrique des comptoirs ou Afrique du développement ?", Le Monde diplomatique, janvier, pp. 24-25.

Reno (William), 1995. Corruption and State Politics in Sierra Leone, Cambridge, Cambridge UP. 
RENO (William), 1997. " War, markets and the reconfiguration of West Africa's weak states », Comparative Politics 29(4), juillet, pp. 493-510.

RENO (William), 1997. "African weak states and commercial alliances », African Affairs, n96, pp. 165-185.

Reno (William), 1998. Warlords Politics and African States, Boulder, Lynne Rienner.

SAINT-Quentin (Grégoire de), 1998. " Mercenariat et mutations stratégiques », Défense nationale, $\mathrm{n}^{\circ} 4$, avril, pp. 34-44.

Shearer (David), 1998. Private Armies and Military Intervention, Adelphi Paper, n³16, Oxford, International Institute for Strategic Studies, 1998.

Thomson (Janice), 1994. Mercenaries, Pirates and Sovereigns. State Building and Extraterritorial Violence in Early Modern Europe, Princeton, Princeton UP.

\section{Pour les informations concrètes, voir}

Chapleau (Philippe) et Misser (François), 1998. Mercenaires S.A., Paris, Desclée De Brouwer.

MISSER (François) et VAlLÉE (Olivier), 1997. Les Gemmocraties. L'économie politique du diamant africain, Paris, Desclée De Brouwer.

ainsi que

Africa Confidential, notamment n³9(5), 6 mars 1998 ; La Lettre du Continent, notamment $n^{\circ} 272,5 / 7 / 1997 ; n^{\circ} 300,26 / 2 / 1998 ; n^{\circ} 310,23 / 7 / 1998$; Jeremy HARDING, « The mercenary business ", London Review of Books, 1/1/1996, pp. 3-8; David SHEARER, « Dial an army : Executive Outcomes in Sierra Leone », The World Today, août-sept. 1997, pp. 203-205 ; "Africa's new look dogs of war ", Weekly Mail and Guardian, 24/1/1997 ; Laurence MAZURE, "Lucrative reconversion des mercenaires sud-africains ", Le Monde diplomatique, oct. 1996, pp. 22-23.

\section{Quelques sites Internet des sociétés de sécurité}

http ://www.elvikingo.com

http ://www.eo.com

http ://www.mpri.com 